\title{
Effect of $\alpha$-tocopherol and $\beta$-carotene supplementation on the incidence of type 2 diabetes
}

\author{
M. Kataja-Tuomola • J. R. Sundell • S. Männistö • \\ M. J. Virtanen • J. Kontto • D. Albanes • J. Virtamo
}

Received: 2 May 2007 / Accepted: 24 September 2007 / Published online: 10 November 2007

(C) Springer-Verlag 2007

\begin{abstract}
Aims/hypothesis Type 2 diabetes is associated with reduced antioxidant defence. Only a few human studies have investigated the role of antioxidants in the pathogenesis of diabetes. This study aimed to examine whether $\alpha$-tocopherol or $\beta$-carotene affected the occurrence of type 2 diabetes.

Methods In the Alpha-Tocopherol, Beta-Carotene Cancer Prevention (ATBC) Study, a double-blind, controlled trial, 29,133 male smokers aged 50-69 years were randomised to receive either $\alpha$-tocopherol $(50 \mathrm{mg} /$ day) or $\beta$-carotene (20 mg/day) or both agents or placebo daily for 5-8 years (median 6.1 years). Baseline serum samples were analysed for $\alpha$-tocopherol and $\beta$-carotene using HPLC. Cases of diabetes were identified from a nationwide Finnish registry of patients receiving drug reimbursement for diabetes. Of 27,379 men without diabetes at baseline, 705 men were diagnosed with diabetes during the follow-up of up to 12.5 years.

Results Baseline serum levels of $\alpha$-tocopherol and $\beta$ carotene were not associated with the risk of diabetes in the placebo group: the relative risk (RR) between the highest and lowest quintiles of $\alpha$-tocopherol was 1.59 (95\% CI 0.89 $2.84)$ and that for $\beta$-carotene was 0.66 (95\% CI $0.40-1.10)$. Neither supplementation significantly affected the incidence of diabetes: the RR was 0.92 (95\% CI 0.79-1.07) for
\end{abstract}

M. Kataja-Tuomola $(\varangle) \cdot$ J. R. Sundell · S. Männistö •

M. J. Virtanen $\cdot$ J. Kontto $\cdot$ J. Virtamo

Department of Health Promotion and Chronic Disease Prevention,

National Public Health Institute,

Mannerheimintie 166,

00300 Helsinki, Finland

e-mail: merja.kataja-tuomola@ktl.fi

\section{Albanes}

Division of Cancer Epidemiology and Genetics,

National Cancer Institute,

Bethesda, MD, USA participants receiving $\alpha$-tocopherol compared with nonrecipients and 0.99 (95\% CI 0.85-1.15) for participants receiving $\beta$-carotene compared with non-recipients.

Conclusions/interpretation Neither $\alpha$-tocopherol nor $\beta$ carotene supplementation prevented type 2 diabetes in male smokers. Serum levels of $\alpha$-tocopherol and $\beta$-carotene were not associated with the risk of type 2 diabetes.

ClinicalTrials.gov ID no. NCT00342992

Keywords $\alpha$-Tocopherol $\cdot \beta$-Carotene - Type 2 diabetes . Randomised controlled trial

\begin{abstract}
Abbreviations
ATBC alpha-tocopherol, beta-carotene cancer prevention $\mathrm{RR}$ relative risk
\end{abstract}

\section{Introduction}

Diabetes is a multifactorial disease that leads to deleterious effects on many organ systems within the body. Oxidative stress mediated by free radicals has been implicated in the pathogenesis of diabetes, and thus antioxidants could protect against diabetes [1]. However, only a few human studies have examined the association between antioxidant status and the risk of type 2 diabetes and the findings have been contradictory [2-6].

The use of vitamin supplements was associated with a $24 \%$ lower risk of diabetes during 20 years of follow-up in the First National Health and Nutrition Examination Survey (NHANES I) Epidemiologic Follow-up Study [7]. The results of controlled trials do not support the hypothesis that antioxidants play a preventive role in the development of type 2 diabetes [8-10]. 
The aim of this study was to examine the effects of supplementary $\alpha$-tocopherol and $\beta$-carotene on the incidence of type 2 diabetes in middle-aged male smokers who participated in the Alpha-Tocopherol, Beta-Carotene Cancer Prevention (ATBC) Study. We also studied the association between baseline serum concentrations of $\alpha$-tocopherol and $\beta$-carotene and the risk of diabetes. Although the primary aim of the ATBC Study was to examine the effects of the supplements on the risk of lung and other cancers, the study design provided a useful basis for evaluating the effects of the supplements on other diseases.

\section{Methods}

Participants The ATBC Study was a randomised, doubleblind, placebo-controlled clinical trial with a $2 \times 2$ factorial design [11]. The participants were screened from among the total male population aged 50-69 years living in southwestern Finland $(n=290,406)$.

A postal survey was performed to identify current smokers willing to participate in the trial. Men who reported smoking five or more cigarettes per day who were willing to participate in the study $(n=42,957)$ were invited to undergo baseline examinations. Exclusion criteria were prior malignancy (other than non-melanoma skin cancer or carcinoma in situ), severe angina on exertion, chronic renal failure, cirrhosis of the liver, alcoholism, anticoagulant therapy, other medical problems that might limit longterm participation, or current use of vitamin E $(>20 \mathrm{mg} /$ day), vitamin $\mathrm{A}[>20,000 \mathrm{IU} /$ day $(>6,000 \mu \mathrm{g} /$ day $)]$ or $\beta$ carotene ( $>6 \mathrm{mg} /$ day) supplements.

A total of 29,133 eligible men were randomly assigned in blocks of eight to one of four intervention groups: $\alpha$ tocopherol (DL- $\alpha$-tocopheryl acetate, $50 \mathrm{mg} /$ day), $\beta$-carotene (20 mg/day), both agents or placebo. Enrolment took place between 1985 and 1988, and the trial intervention continued until 30 April 1993. Active intervention ranged from 5 to 8 years (median 6.1 years).

The institutional review boards of the National Public Health Institute, Finland, and the National Cancer Institute, USA, approved the ATBC Study. All participants provided their written, informed consent before randomisation.

Data collection At baseline, data on background characteristics such as medical and dietary histories, smoking, alcohol consumption and physical activity were collected by questionnaire and checked by specially trained registered nurses. Use of alcohol during the previous year was assessed by enquiring about the amount and type (beer, wine, spirit) consumed, and the mean daily intake of pure ethanol in grams was calculated. Leisure-time physical activity during the past year was assessed as sedentary (e.g. reading, watching television), moderate (e.g. walking, hunting, gardening) and heavy (e.g. running, skiing, swimming). Height and weight were measured, and the BMI was calculated. Blood pressure was measured by mercury sphygmomanometry of the right arm while the participant remained seated. The lower of two measurements taken at least $1 \mathrm{~min}$ apart was recorded.

A blood sample was drawn and the serum was stored at $-70^{\circ} \mathrm{C}$. Serum cholesterol concentrations were determined enzymatically [CHOD-PAP (cholesterol oxidase-phenol ampyrone) method; Boehringer Mannheim, Mannheim, Germany]. HDL-cholesterol was measured after precipitation with dextran sulphate and magnesium chloride. Serum concentrations of $\alpha$-tocopherol and $\beta$-carotene were determined by high-performance liquid chromatography [12]. The between-run coefficients of variation were $2.2 \%$ for $\alpha$ tocopherol and $3.6 \%$ for $\beta$-carotene. Serum glucose was determined by the enzymatic hexokinase method using an Optima analyser (ThermoFischer, Vantaa, Finland).

The participants made a follow-up visit to local field centres three times each year. During each visit they returned their remaining study capsules and received a new supply. Capsule compliance (median $99 \%$ of on-study capsules taken) and the dropout rate (31\%) were similar across the four intervention groups [13]. A follow-up serum sample was taken 3 years after study entry and analysed for $\alpha$-tocopherol and $\beta$-carotene.

The metabolic syndrome was defined by means of BMI, blood pressure, and HDL-cholesterol according to the criteria set by the International Diabetes Federation [14]: BMI $>30 \mathrm{~kg} / \mathrm{m}^{2}$, physician-diagnosed hypertension or systolic blood pressure $\geq 130 \mathrm{mmHg}$ or diastolic blood pressure $\geq 85 \mathrm{mmHg}$, and HDL-cholesterol $<1.03 \mathrm{mmol} / \mathrm{l}$. Indicators for insulin resistance or the prothrombic or proinflammatory state were unavailable.

Assessment of diabetes In Finland, all patients requiring prescription treatment for diabetes are entitled to reimbursement of their medication expenses. This requires a detailed medical certificate from the attending physician. The Social Insurance Institution checks that the case fulfils the criteria set for diabetes and maintains a register of these cases. The ATBC study participants were linked to the register through the unique personal identity number assigned to each Finnish citizen. At study entry, 1,272 participants were entitled to reimbursement of diabetes medication, or they reported receiving diabetes medication or having physician-diagnosed, diet-treated diabetes. Of the incident cases up to December 1997, 482 had a high concentration of serum glucose (i.e. $\geq 7.0 \mathrm{mmol} / \mathrm{l}$ ) in the baseline serum sample. After exclusion of these cases, the final cohort for this study comprised 27,379 men, among whom 705 incident cases of diabetes with baseline serum glucose below $7.0 \mathrm{mmol} / \mathrm{l}$ were identi- 
Fig. 1 Study design and participants

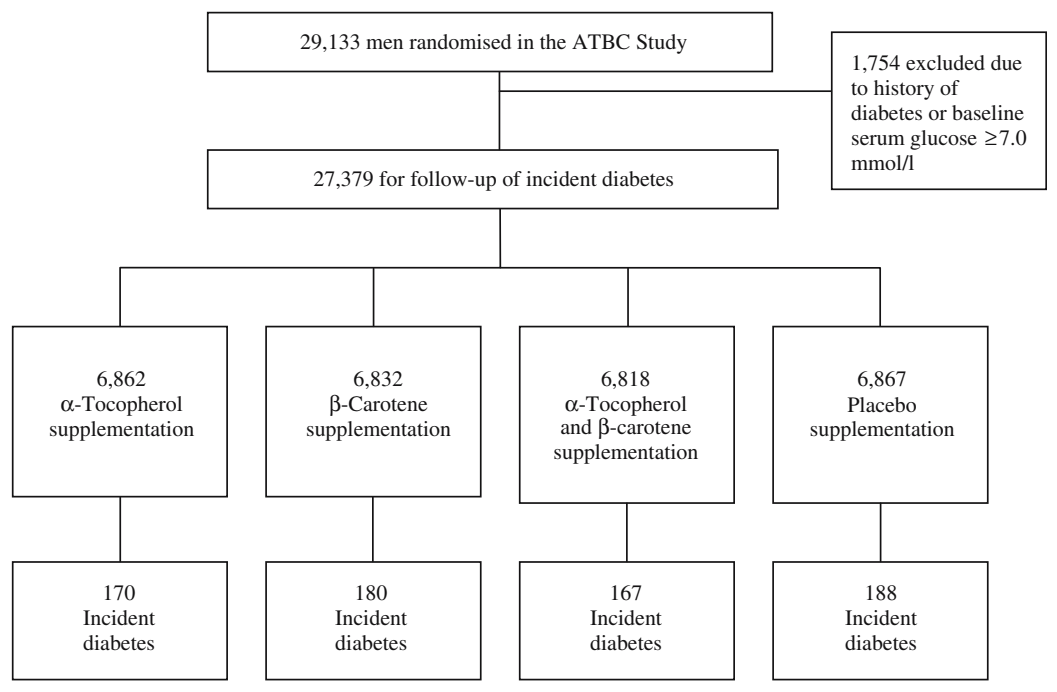

fied from the drug reimbursement register up to December 1997 (Fig. 1). Of these incident cases, 305 men were entitled to drug reimbursement during the intervention period by April 1993.

We also analysed a random sample of 500 non-cases of the final cohort for baseline serum glucose. In this analysis, the serum glucose concentration in ten non-cases was $7.0 \mathrm{mmol} / \mathrm{l}$ or higher; in seven men it varied from 7.07 to $7.53 \mathrm{mmol} / \mathrm{l}$ and in only three men did it exceed the drug reimbursement criterion of $8.0 \mathrm{mmol} / \mathrm{l}$.

Statistical analysis All analyses were by intention to treat. The intervention-specific cumulative incidence of diabetes was calculated by the Kaplan-Meier method using the log-rank test to calculate the statistical significance of differences between intervention groups. The effect of intervention was estimated by Cox proportional hazards regression and expressed as the relative risk (RR) and its $95 \% \mathrm{CI}$. We present the effects for each supplementation separately after testing for interaction between $\alpha$-tocopherol and $\beta$-carotene: $\alpha$-tocopherol compared with no $\alpha$-tocopherol, and $\beta$-carotene compared with no $\beta$ carotene. We tested the proportional hazards assumption with no evidence of non-proportional hazards (for $\alpha$-tocopherol vs no $\alpha$-tocopherol, $p=0.14$; for $\beta$-carotene vs no $\beta$-carotene, $p=0.82$ ) [15]. The follow-up time extended from the date of randomisation until entitlement to drug reimbursement for diabetes, death or the end of follow-up (i.e. December 1997), with a total of 261,868 person-years. The effect of each supplementation during the follow-up period was expressed by calculating relative risks and $95 \%$ CIs within consecutive time intervals each containing 30 cases of diabetes and plotting the estimates using 'super smoother' [16].

The likelihood ratio test was used to study whether baseline metabolic syndrome modified the effects of $\alpha$ tocopherol and $\beta$-carotene on diabetes incidence by comparing models with and without the cross-product term.
Table 1 Baseline characteristics of the cohort for follow-up of incident diabetes in the four intervention groups

Data are median or percentage Alcohol consumption data were missing for $6.8 \%$ of participants; other characteristics were missing for $0-0.2 \%$

\begin{tabular}{lllll}
\hline Characteristic & $\alpha$-Tocopherol & $\beta$-Carotene & $\begin{array}{l}\alpha \text {-Tocopherol } \\
\text { and } \beta \text {-carotene }\end{array}$ & Placebo \\
& & & 6,818 & 6,867 \\
\hline Number of participants & 6,862 & 6,832 & 57.3 & 56.8 \\
Age (years) & 57.1 & 57.2 & 20 & 20 \\
Number of cigarettes per day & 20 & 20 & 36 & 36 \\
Smoking (years) & 36 & 37 & 6.19 & 6.15 \\
Total cholesterol (mmol/l) & 6.15 & 6.16 & 1.15 & 1.16 \\
HDL-cholesterol (mmol/1) & 1.15 & 1.16 & 25.9 & 25.8 \\
BMI (kg/m ${ }^{2}$ ) & 25.8 & 25.8 & 140 & 140 \\
Systolic blood pressure (mmHg) & 140 & 140 & 88 & 88 \\
Diastolic blood pressure (mmHg) & 88 & 88 & 11 & 11 \\
Alcohol consumption (g/day) & 11 & 11 & 59 & 58 \\
Moderate or heavy leisure-time & 59 & 58 & & \\
physical activity (\%) & & & \\
\hline
\end{tabular}


The associations between quintiles of baseline serum concentrations of $\alpha$-tocopherol and $\beta$-carotene and the incidence of diabetes were calculated by Cox regression analysis in the placebo group. These analyses were adjusted for the continuous variables age, BMI, number of daily cigarettes, years of smoking, serum total cholesterol, HDLcholesterol, systolic and diastolic blood pressures, alcohol intake and leisure-time physical activity. All analyses were carried out with the R statistical programme [17].

\section{Results}

At baseline, the median age of the participants was 57 years, they smoked a median of 20 cigarettes daily at that time, and their median BMI was $26 \mathrm{~kg} / \mathrm{m}^{2}$. We found no differences in background characteristics between the intervention groups at baseline (Table 1).

Serum concentrations of $\alpha$-tocopherol increased from a median of $26.8 \mu \mathrm{mol} / 1$ at baseline to $40.2 \mu \mathrm{mol} / 1$ at 3 years in the $\alpha$-tocopherol-supplemented group. Similarly, $\beta$-carotene supplementation increased serum $\beta$-carotene concentrations from a median of $0.34 \mu \mathrm{mol} / \mathrm{l}$ at baseline to $5.60 \mu \mathrm{mol} / 1$ at 3 years. In the non-supplemented $\alpha$-tocopherol and $\beta$ carotene groups, we observed no change in serum concentrations of $\alpha$-tocopherol and $\beta$-carotene, respectively, during 3 years.

The incidence of diabetes per 1,000 person-years varied from 2.6 to 2.8 in the intervention groups (Table 2). The differences in incidence between the groups were not statistically significant (log-rank test, $1.34 ; p=0.72$ ). No interaction was found between $\alpha$-tocopherol and $\beta$-carotene supplementation $(p=0.85)$.

In the $2 \times 2$ factorial comparison, no statistically significant difference in risk of diabetes was observed between $\alpha$-tocopherol recipients and non-recipients (RR 0.92; 95\%

Table 2 Incidence and relative risk of diabetes in the four intervention groups

\begin{tabular}{lllll}
\hline $\begin{array}{l}\text { Intervention } \\
\text { group }\end{array}$ & $\begin{array}{l}\text { Number } \\
\text { of cases }\end{array}$ & $\begin{array}{l}\text { Person- } \\
\text { years }\end{array}$ & $\begin{array}{l}\text { Incidence } \\
\text { per 1,000 } \\
\text { person-years }\end{array}$ & $\begin{array}{l}\text { Relative } \\
\text { risk } \\
(95 \% \mathrm{CI})\end{array}$ \\
\hline$\alpha$-Tocopherol & 170 & 65,822 & 2.6 & $\begin{array}{c}0.91 \\
(0.73-1.12)\end{array}$ \\
$\beta$-Carotene & 180 & 65,151 & 2.8 & $\begin{array}{c}0.97 \\
(0.79-1.20)\end{array}$ \\
$\begin{array}{l}\alpha \text {-Tocopherol } \\
\text { and } \beta \text {-carotene }\end{array}$ & 167 & 64,892 & 2.6 & $\begin{array}{c}0.91 \\
(0.73-1.12)\end{array}$ \\
$\begin{array}{l}\text { Placebo } \\
188\end{array}$ & 66,003 & 2.8 & $\begin{array}{c}1.00 \\
\text { (reference) }\end{array}$ \\
\hline
\end{tabular}

CI $0.79-1.07)$ or between $\beta$-carotene recipients and nonrecipients (RR 0.99; 95\% CI 0.85-1.15). When excluding cases occurring during the first 2 years of follow-up the RR was $0.91(95 \%$ CI $0.78-1.06)$ for $\alpha$-tocopherol and 0.99 (95\% CI $0.85-1.16)$ for $\beta$-carotene. When including only cases from the intervention period (up to April 1993) the RR was 0.97 (95\% CI $0.77-1.22)$ for $\alpha$-tocopherol and 1.01 (95\% CI $0.80-1.26)$ for $\beta$-carotene.

The relative risk of diabetes varied non-significantly around unity with follow-up time both for $\alpha$-tocopherol recipients compared with non-recipients and for $\beta$-carotene recipients compared with non-recipients (Fig. 2).

Baseline metabolic syndrome did not modify the effect of $\alpha$-tocopherol or $\beta$-carotene supplementation on the incidence of diabetes ( $p$ for interaction, 0.40 and 0.29 respectively).

There was no significant difference in the risk of diabetes between the quintiles of baseline serum $\alpha$-tocopherol or $\beta$ carotene concentration (Table 3). When the four highest quintiles of serum $\beta$-carotene were combined, a decreased risk of diabetes was seen compared with the lowest quintile (RR $0.70 ; 95 \%$ CI $0.49-0.99$ ). The effect of supplementary $\beta$-carotene was similar in the four highest quintiles combined and in the lowest quintile of serum $\beta$-carotene ( $p$ for interaction 0.12 ).
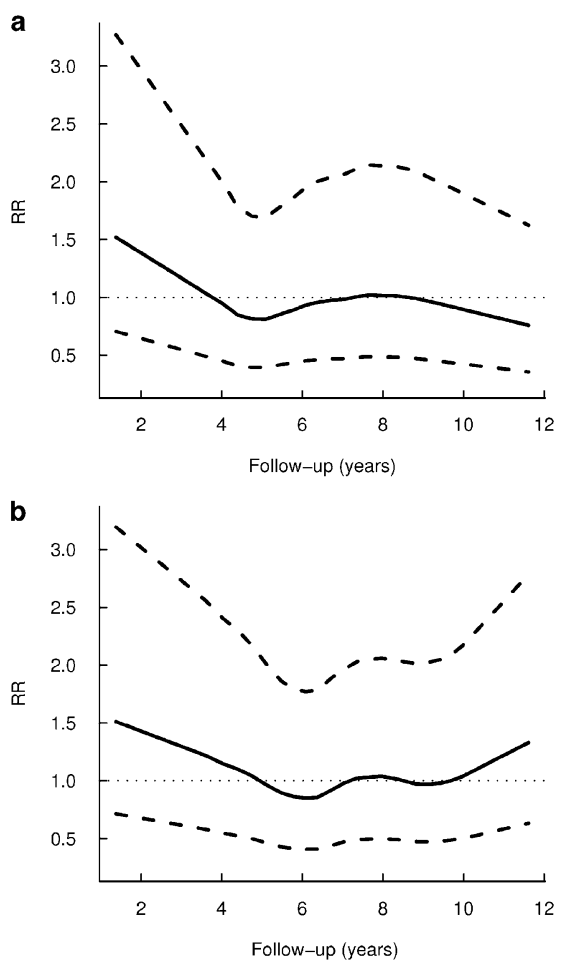

Fig. 2 RR of diabetes with $95 \%$ CIs for a $\alpha$-tocopherol vs no- $\alpha$ tocopherol and $\mathbf{b} \beta$-carotene vs no- $\beta$-carotene supplementation with follow-up time 


\section{Discussion}

We found no effect of supplementary $\alpha$-tocopherol on the incidence of type 2 diabetes in middle-aged male smokers. This is in keeping with the outcomes of other controlled trials. In the Heart Outcomes Prevention Evaluation (HOPE) Trial, 124 participants supplemented with a natural source of vitamin E (400 IU; $R R R$ - $\alpha$-tocopheryl acetate, $294 \mathrm{mg}$ ) daily and 137 participants taking placebo reported incident diabetes during an average follow-up of 4.5 years $(p=0.55)$ [8]. Recently the Women's Health Study reported that 827 incident cases of type 2 diabetes occurred in the vitamin $\mathrm{E}$ group (600 IU $\alpha$-tocopherol every other day for 10 years) and 869 in the placebo group (RR 0.95 ; 95\% CI $0.87-1.05$ ) [9]. In our study, supplementation with $\beta$-carotene had no effect on diabetes incidence. This finding is consistent with the Physicians' Health Study, a placebo-controlled trial of supplementation with $50 \mathrm{mg} \beta$-carotene on alternate days for 12 years, in which 396 men reported type 2 diabetes in the $\beta$-carotene group and 402 men did so in the placebo group (RR 0.98\%; 95\% CI 0.85-1.12) [10]. A combination of $120 \mathrm{mg}$ vitamin $\mathrm{C}, 30 \mathrm{mg}$ vitamin $\mathrm{E}, 6 \mathrm{mg} \beta$-carotene, $100 \mu \mathrm{g}$ selenium and $20 \mathrm{mg}$ zinc daily for 7.5 years had no effect on fasting plasma glucose [18].

Our findings on the baseline serum concentrations of $\alpha$ tocopherol and $\beta$-carotene were consistent with those regarding supplementation: baseline concentrations of $\alpha$ tocopherol and $\beta$-carotene were not associated with the risk of diabetes. The lower risk of diabetes in the four highest quintiles combined compared with the lowest quintile of serum $\beta$-carotene concentration was probably due to residual confounding. These findings contradict the results of two prospective observational studies, from Finland and the USA, in which a low concentration of plasma vitamin $\mathrm{E}$ was associated with excess risk of type 2 diabetes [4, 5]. In a Finnish prospective study, the inverse association between serum $\alpha$-tocopherol and $\beta$-carotene and the incidence of diabetes disappeared after adjustment for cardiovascular risk factors [3]. The numbers of incident cases of diabetes in these three studies were small, however, ranging from 45 to 106 . A recent study among US women found no prospective association between baseline plasma $\beta$-carotene and the risk of type 2 diabetes, based on 470 incident cases of diabetes [6].

Certain hypotheses suggest that vitamin E could potentially influence insulin sensitivity. The antioxidant property of vitamin $\mathrm{E}$ is often considered a key activity, although vitamin E does cause other effects that could potentially modify the action of insulin. For example, vitamin $\mathrm{E}$ inhibits protein kinase $\mathrm{C}$ activity, which has been associated with insulin resistance experimentally [19]. Vitamin E regulates several genes; for example, it upregulates the expression of $P P A R G$, the gene for peroxisome proliferator activated receptor $\gamma$ $(\operatorname{PPAR} \gamma)$ [20]. PPAR $\gamma$ is a nuclear receptor the stimulation of which improves glucose tolerance and insulin sensitivity in type 2 diabetes mellitus patients and in animal models of insulin resistance [21]. Vitamin $\mathrm{E}$ possesses a structural similarity to glucose-lowering agents such as thiazolidinediones, which are agonists for PPAR $\gamma$.

$\beta$-Carotene as an antioxidant has been hypothesised to provide protection against diabetes [22]. Although we found that supplementation with $\alpha$-tocopherol and $\beta$ -

Table 3 Relative risk (RR) and 95\% CI of diabetes by quintiles of baseline serum $\alpha$-tocopherol and $\beta$-carotene in the placebo group

\begin{tabular}{|c|c|c|c|c|c|c|c|}
\hline \multirow[t]{2}{*}{ Serum factor } & \multicolumn{5}{|l|}{ Quintiles } & \multirow{2}{*}{$\begin{array}{l}p \text { value for } \\
\text { homogeneity }\end{array}$} & \multirow{2}{*}{$\begin{array}{l}p \text { value for } \\
\text { trend }\end{array}$} \\
\hline & 1 & 2 & 3 & 4 & 5 & & \\
\hline \multicolumn{8}{|l|}{$\alpha$-Tocopherol (mg/l) } \\
\hline Median & 8.30 & 10.11 & 11.40 & 12.90 & 15.70 & & \\
\hline Range & $0.14-9.34$ & $9.35-10.77$ & $10.78-12.09$ & $12.10-13.97$ & $13.98-39.71$ & & \\
\hline Number of cases & 29 & 36 & 35 & 33 & 55 & & \\
\hline Person-years & 12,673 & 13,267 & 13,091 & 13,664 & 13,274 & & \\
\hline Multivariate $\mathrm{RR}^{\mathrm{a}}$ & 1.00 & 1.09 & 1.24 & 1.07 & 1.59 & & \\
\hline $95 \% \mathrm{CI}$ & & $0.65-1.82$ & $0.72-2.11$ & $0.61-1.88$ & $0.89-2.84$ & 0.41 & 0.16 \\
\hline \multicolumn{8}{|l|}{$\beta$-Carotene $(\mu \mathrm{g} / \mathrm{l})$} \\
\hline Median & 72 & 125 & 174 & 241 & 379 & & \\
\hline Range & $0-101$ & $102-147$ & $148-203$ & 204-292 & $293-5445$ & & \\
\hline Number of cases & 51 & 42 & 33 & 35 & 27 & & \\
\hline Person-years & 12,472 & 12,974 & 13,265 & 13,573 & 13,686 & & \\
\hline Multivariate $\mathrm{RR}^{\mathrm{a}}$ & 1.00 & 0.74 & 0.65 & 0.73 & 0.66 & & \\
\hline $95 \% \mathrm{CI}$ & & $0.49-1.14$ & $0.41-1.02$ & $0.46-1.16$ & $0.40-1.10$ & 0.34 & 0.11 \\
\hline
\end{tabular}

${ }^{\text {a }}$ RR adjusted for the continuous variables age, BMI, number of cigarettes per day, years of smoking, total cholesterol, HDL-cholesterol, systolic and diastolic blood pressures, alcohol consumption and leisure-time physical activity 
carotene had no effect on the risk of diabetes, it is possible that there was no effect because the intervention period was too short or the timing of the intervention was wrong. Also, the findings do not exclude the possibility that other antioxidants might protect against type 2 diabetes.

Our study had several strengths. The randomisation resulted in even distributions of baseline characteristics across intervention groups. The successful supplementation was evidenced by high capsule compliance and substantial increases in serum $\alpha$-tocopherol and $\beta$-carotene concentrations in the respective supplementation groups.

We were able to control the baseline serum glucose level in the register-based incident cases of diabetes and thus to exclude cases who had elevated serum glucose indicating the presence of diabetes at baseline. We also determined baseline serum glucose concentrations in a random sample of 500 non-cases and found that ten men in this analysis had serum glucose levels of $7.0 \mathrm{mmol} / \mathrm{l}$ or higher. Extrapolating from this, about 530 non-cases had elevated baseline serum glucose. Since as a result of randomisation they were equally distributed to the supplementation groups, their identification and exclusion would have increased the incidence rates only by up to 0.06 per 1,000 person-years or $2.2 \%$. Thus, their inclusion in the study population cannot explain our finding of no effect.

We retrieved cases of incident diabetes from a nationwide register of drug reimbursements. The criteria for reimbursement for diabetes drugs required a fasting whole blood glucose concentration of $7.0 \mathrm{mmol} / \mathrm{l}$ or higher or a fasting plasma glucose concentration of $8.0 \mathrm{mmol} / \mathrm{l}$ or higher. Thus, the register identified only the more advanced cases of diabetes. Our study is therefore not informative regarding diabetes cases with fasting whole blood glucose between 6.1 and $6.9 \mathrm{mmol} / 1$ or plasma glucose between 7.0 and $7.9 \mathrm{mmol} / \mathrm{l}$, as defined by the WHO-99 criteria [23].

In a Finnish study in which participants were screened for diabetes with an oral glucose tolerance test, $13.6 \%$ of men aged 55-64 years had prevalent diabetes based on WHO-99 criteria, and $44 \%$ of these were unaware of their diabetic condition [24]. We do not have data to estimate how many of the ATBC Study participants might have had undiagnosed diabetes fulfilling the criteria of drug reimbursement at the end of follow-up in December 1997. There is, however, no reason to suppose that supplementation had influenced differently the diagnosis of diabetes, and thus the undiagnosed cases fulfilling the criteria of drug reimbursement are unlikely to affect our risk estimates of the effect of supplementation.

The register provides no information about whether the diabetes is type 1 or type 2 . In a Finnish survey, $96 \%$ of all diabetic participants diagnosed after the age of 55 years had type 2 diabetes [25]. Participants in our study were 5069 years of age at study entry, and thus the incident cases of diabetes represent primarily type 2 diabetes.
Our study included only older middle-aged male smokers since the primary aim of the ATBC Study was to examine the preventive effects of $\alpha$-tocopherol and $\beta$ carotene on lung cancer. Therefore, our results cannot be generalised to non-smokers and females. This is especially noteworthy since smoking increases the risk of diabetes. In a 12-year prospective cohort study of 275,190 men, the rate of diabetes was $45 \%$ higher among those who smoked at least two packs daily at baseline than among those who had never smoked [26]. In a 6-year follow-up study of 41,810 health professionals, the relative risk of diabetes among men who smoked at least 25 cigarettes daily was $1.94(95 \%$ CI, 1.25-3.03) times higher than that of non-smokers [27].

In conclusion, supplementation with $\alpha$-tocopherol or $\beta$ carotene had no preventive effect on the risk of type 2 diabetes in middle-aged male smokers. Baseline serum levels of $\alpha$-tocopherol and $\beta$-carotene were also not associated with the risk of diabetes. However, since our endpoint assessment identified diabetes cases requiring drug treatment, the possible roles of $\alpha$-tocopherol and $\beta$ carotene in the development of early insulin resistance remain open and require further study.

Acknowledgements This ATBC Study was supported by US Public Health Service contracts N01-CN-45165, N01-RC-45035 and N01RC-37004 from the National Cancer Institute, National Institutes of Health and the Department of Health and Human Services.

Duality of interest The authors declare that there is no duality of interest associated with this manuscript.

\section{References}

1. Jennings PE, Jones AF, Florkowski CM, Lunec J, Barnett AH (1987) Increased diene conjugates in diabetic subjects with microangiopathy. Diabet Med 4:452-456

2. Montonen J, Knekt P, Jarvinen R, Reunanen A (2004) Dietary antioxidant intake and risk of type 2 diabetes. Diabetes Care 27:362-366

3. Reunanen A, Knekt P, Aaran RK, Aromaa A (1998) Serum antioxidants and risk of non-insulin dependent diabetes mellitus. Eur J Clin Nutr 52:89-93

4. Salonen J, Nyyssonen K, Tuomainen TP et al (1995) Increased risk of non-insulin dependent diabetes mellitus at low plasma vitamin E concentrations: a four year follow up study in men. BMJ 311:1124-1127

5. Mayer-Davis EJ, Costacou T, King I, Zaccaro DJ, Bell RA (2002) Plasma and dietary vitamin $\mathrm{E}$ in relation to incidence of type 2 diabetes: the Insulin Resistance and Atherosclerosis Study (IRAS). Diabetes Care 25:2172-2177

6. Wang L, Liu S, Pradhan AD et al (2006) Plasma lycopene, other carotenoids, and the risk of type 2 diabetes in women. Am J Epidemiol 164:576-585

7. Ford ES (2001) Vitamin supplement use and diabetes mellitus incidence among adults in the United States. Am J Epidemiol 153:892-897 
8. Lonn E, Yusuf S, Hoogwerf B et al (2002) Effects of vitamin E on cardiovascular and microvascular outcomes in high-risk patients with diabetes: results of the HOPE Study and MICRO-HOPE Substudy. Diabetes Care 25:1919-1927

9. Liu S, Lee I, Song Y et al (2006) Vitamin E and risk of type 2 diabetes in the Women's Health Study randomized controlled trial. Diabetes 55:2856-2862

10. Liu S, Ajani U, Chae C, Hennekens C, Buring JE, Manson JE (1999) Long-term beta-carotene supplementation and risk of type 2 diabetes mellitus: a randomized controlled trial. JAMA 282: 1073-1075

11. The ATBC Cancer Prevention Study Group (1994) The AlphaTocopherol, Beta-Carotene Lung Cancer Prevention Study: design, methods, participant characteristics and compliance. Ann Epidemiol $4: 1-10$

12. Milne DB, Botnen J (1986) Retinol, alpha-tocopherol, lycopene, and alpha-and beta-carotene simultaneously determined in plasma by isocratic liquid chromatography. Clin Chem 32:874-876

13. The Alpha-Tocopherol, Beta-Carotene Cancer Prevention Study Group (1994) The effect of vitamin E and beta-carotene on the incidence of lung cancer and other cancers in male smokers. $\mathrm{N}$ Engl J Med 330:1029-1035

14. Alberti KG, Zimmet P, Shaw J (2006) Metabolic syndrome-a new world-wide definition. A consensus statement from the International Diabetes Federation. Diabet Med 23:469-480

15. Grambsch P, Therneau T (1994) Proportional hazards tests and diagnostics based on weighted residuals. Biometrika 81:515-526

16. Friedman JH (1984) SMART user's guide. Stanford University Technical Report No. 1. Laboratory for Computational Statistics, Stanford University, Palo Alto

17. Ihaka R, Gentleman R (1996) A language for data analysis and graphics. J Comput Graph Stat 5:299-314
18. Czernichow S, Couthouis A, Bertrais S et al (2006) Antioxidant supplementation does not affect fasting plasma glucose in the Supplementation with Antioxidant Vitamins and Minerals (SU.VI. MAX) Study in France: association with dietary intake and plasma concentrations. Am J Clin Nutr 84:395-399

19. Griffin ME, Marcucci MJ, Cline GW et al (1999) Free fatty acidinduced insulin resistance is associated with activation of protein kinase $\mathrm{C}$ theta and alterations in the insulin signaling cascade. Diabetes 48:1270-1274

20. Azzi A, Gysin R, Kempna P et al (2004) Regulation of gene expression by alpha-tocopherol. Biol Chem 385:585-591

21. Picard F, Auwerx J (2002) PPAR(gamma) and glucose homeostasis. Annu Rev Nutr 22:167-197

22. Maritim AC, Sanders RA, Watkins III JB (2003) Diabetes, oxidative stress, and antioxidants: a review. J Biochem Mol Toxicol 17:24-38

23. World Health Organization (1999) Definition, diagnosis and classification of diabetes mellitus and its complications. Part I. Diagnosis and classification of diabetes mellitus. Report of a WHO Consultation. Department of Noncommunicable Disease Surveillance, World Health Organization, Geneva

24. Ylihärsilä H, Lindström J, Eriksson JG et al (2005) Prevalence of diabetes and impaired glucose regulation in 45- to 64-year-old individuals in three areas of Finland. Diabet Med 22:88-91

25. Laakso M, Pyörälä K (1985) Age of onset and type of diabetes. Diabetes Care 1985(8):114-117

26. Will J, Galuska A, Ford E, Mokdad A, Calle E (2001) Cigarette smoking and diabetes mellitus: evidence of positive association from a large prospective cohort study. Int J Epidemiol 30:540-546

27. Rimm E, Chan J, Stampfer MJ, Colditz GA, Willett WC (1995) Prospective study of cigarette smoking, alcohol use, and the risk of diabetes in men. Br Med J 310:555-559 